\title{
Automatic reconstruction of polycrystalline ice microstructure from image analysis: application to the EPICA ice core at Dome Concordia, Antarctica
}

\author{
Michel Gay, Jerome Weiss \\ Laboratoire de Glaciologie et Géophysique de l'Environnement du CNRS, BP 96, 38402 Saint-Martin-d'Hères Cedex, France
}

\begin{abstract}
A digital image-processing approach is proposed which allows the extraction of two-dimensional polycrystalline ice microstructure (grain boundaries) from thin sections observed between cross-polarisers. It is based on image segmentation of colour images. The method is applied to the preliminary analysis of the shallow (Holocene) ice of the European Project for Ice Coring in Antarctica (EPICA) ice core at Dome Concordia. Structural parameters, such as the mean cross-sectional area, shape anisotropy and grain morphology, are obtained. The interest and limitations of this automatic procedure are discussed.
\end{abstract}

\section{INTRODUGTION}

As with many crystalline materials, ice microstructure, i.e. the size and morphology of grains, controls many physical and mechanical properties of the material. It is therefore very important to obtain as complete as possible a description of this microstructure. This includes not only mean grain-size measurements (we will come back later to the definition of the "size"), but also size distribution, shape anisotropy (elongation of grains along a preferential direction), grain morphology (through the volume/surface or surface/ perimeter relations) and topological characteristics. Moreover, as stressed by Alley and others (1986) and Petit and others (1987), ice microstructure may record the age and past history of ice. Indeed, microstructure evolution depends on strain history, temperature and impurity content (Alley and others, 1986; Alley and Woods, 1996), parameters which vary with climate. This is of particular importance when studying polar ice from deep ice cores. For example, correlations have been observed between climatic record from $\delta^{18} \mathrm{O}$ isotopic studies and mean grain-size evolution in polar ice (Duval and Lorius, 1980; Thorsteinsson and others, 1995). Correlations between grain-size and impurity content, though relatively noisy, have also been reported (Thorsteinsson and others, 1995; Alley and Woods, 1996).

Most of the analyses of ice microstructure presented so far were performed manually on two-dimensional thin sections. They were therefore laborious and time-consuming. For this reason, most of these analyses were restricted to the study of mean grain-size evolution. Several different methods were used to estimate this "size". Gow (1969), in polar firn, measured the length and breadth of the 50 largest crystals in each section with a "pocket comparator". These 50 crystals represented at least $25 \%$ of the total number of crystals, but this representativity depended on the number of crystals in the section and therefore on the mean grainsize itself. These crystals do not therefore represent the entire population of grains, especially if size distribution changes with time. Duval and Lorius (1980) estimated mean grain-size from crystal-counting on a given area, but did not take into account the smallest grains, sometimes of ambiguous existence. Once again, the entire population is not taken into account. Thorsteinsson and others (1995) and Alley and Woods (1996) used the linear intercept method. Alley and Woods (1996) reported some data about size distributions as well. Although probably less "subjective" than the others, this method can raise some problems of interpretation. The mean intercept length is related to the mean cross-sectional area through the average morphology of grains which can change with time. Moreover, Thorsteinsson and others (1995) performed linear intercept analysis along a unique (vertical) direction, which is not representative of real grain morphology if grains are not isotropic and if this shape anisotropy is changing (as observed during this work; see below). The definition of grain "size" is actually not trivial. As demonstrated in this work, different size-estimation methods can lead to very different results (e.g. for graingrowth kinetics; see below), and can therefore be misleading. Another problem with manual methods is related to "human" bias. The acuity, concentration and measuring ability of a worker can vary greatly during a long, tiring day, especially in a cold environment. This will probably increase the discrepancy of results.

In order to obtain the most complete representation of ice microstructure on two-dimensional thin sections, all the grain boundaries have to be extracted. It is worth noting here that this complete description is necessary (though perhaps not sufficient) to reconstitute three-dimensional microstructure from two-dimensional analysis (Underwood, 1970). This cannot be reasonably achieved manually. Here, we present an automatic method, based on image analysis, which allows easy and rapid extraction of ice microstructure from thin sections examined between crossed polarisers. After a technical presentation of the method and the image-analysis procedure, we illustrate its interest with a preliminary study of ice microstructure in 
the shallow part (Holocene ice) of the European Project for Ice Coring in Antarctica (EPICA) ice core at Dome C, discussing the definition of grain "size", shape anisotropy and grain morphology.

\section{EXPERIMENTAL PROCEDURE}

The method of extraction of grain boundaries from colour images of two-dimensional thin sections is based on image segmentation from variation of colour between neighbouring grains observed between crossed polarisers. Arnaud and others (1998) presented a different technique to reveal and automatically characterise pores and grain boundaries in firn, based on sample sublimation, coaxial episcopy and image analysis. Using this technique, Arnaud (1997) determined distributions of grain cross-sectional areas at different depths in firn at Vostok station, Antarctica. Though worth using to study porous media (e.g. firn), optimal sublimation conditions are not easy to obtain, and hence sample preparation is difficult and time-consuming. The "standard" thin sections used in the present work are easily and rapidly obtained. Eicken (1993) proposed an automated image analysis of ice thin sections under crossed polarisers. Unlike the present work, this author used a universal (Rigsby) stage and grey-scale digitisation.

\section{Preparation of thin sections and image recording}

Ice thin sections are prepared by regular microtoming. Section thickness is an important parameter. The optimised thickness is a compromise between large enough colour differences between grains, and sharp enough colour transitions. Too thick sections have great colour differences, but grain boundaries appear too thick, with coloured interference fringes which can be misleading for image analysis. Too thin sections have well-defined spatial transitions but weak differences, most of the grains appearing within a grey range of colours. Experience shows that the optimised thickness corresponds to grain colours around a brown-yellow range. An example is given in Figure la. Note that in the case of examination of columnar ice, with column axis perpendicular to the section, this thickness problem would be less crucial, grain boundaries being almost perpendicular to the section.

For each thin section, three different pictures are taken while crossed polarizers are rotated together $\left(\right.$ at $0^{\circ}, 30^{\circ}$ and $60^{\circ}$ ), the thin section itself being fixed with respect to the camera. In this way, grains remaining dark for one rotation angle are illuminated for other angles, except if their $c$ axes are perpendicular to the section. The image fields must coincide for the three different pictures. Note that a universal stage is not used in the present analysis. For the analysis of the EPICA ice core (see below), slides were taken with a regular camera, then digitalised using a charge coupled device colour camera. However, digital image recording can be performed directly with a digital camera. The format of the images was $640 \times 480$ pixels. The chosen image field resulted from a compromise between a good resolution and a reasonable statistical population of grains, i.e. at least 200. In the study of the shallow part of the EPICA ice core, with mean cross-sectional area of grains ranging between 1 and $2 \mathrm{~mm}^{2}$, this corresponded to a resolution of $42.5 \mu \mathrm{m} /$ pixel. The sampled population of grains varied from about 500 grains at $100 \mathrm{~m}$ depth to slightly less than 200 grains at $360 \mathrm{~m}$.

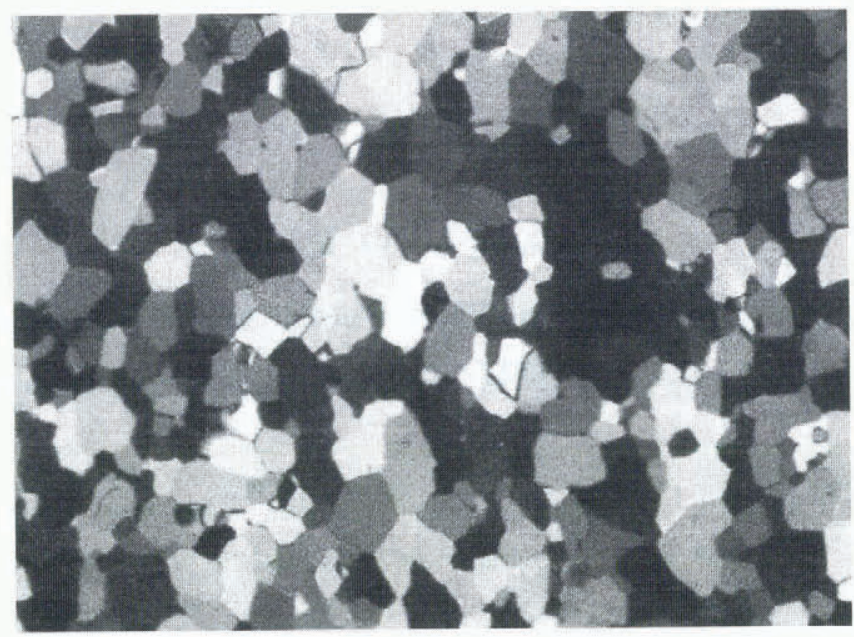

a $\quad 1 \mathrm{~cm}$

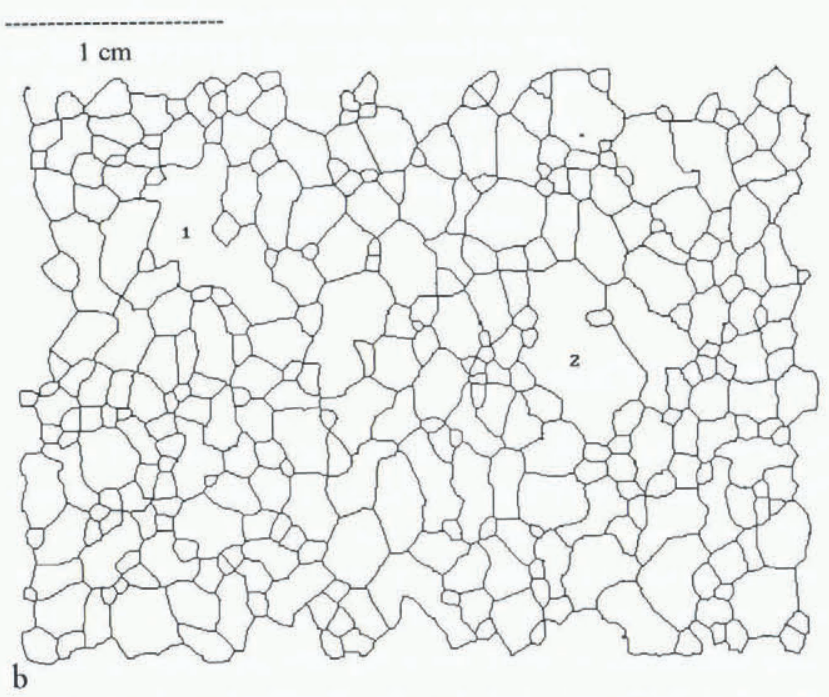

Fig. 1. (a) Thin section of polar ice examined between crossed polarisers, with an optimised thickness for image analysis, EPICA ice core, Dome $C$ (depth $170 \mathrm{~m}$ ). (b) The corresponding binary image of the microstructure (grain boundaries), after digital image processing. 1 and 2 are regions where the segmentation of grains was difficult, owing to $\mathrm{c}$ axes of grains perpendicular to the thin section.

\section{Image analysis}

Image segmentation aims to subdivide the image into regions of homogeneous colour characteristics (Gonzales and Woods, 1992). The discontinuities, i.e. grain boundaries in the present case, correspond to sharp spatial variations of these characteristics. They can be detected using derivative operators (or filters; Gonzales and Woods, 1992). However, pre-processing is generally required in order to remove sharp but small variations resulting from noise, while preserving large variations. Therefore, any image-segmentation processing should include a pre-processing step in order to remove noise and sharpen variations, followed by detection of the discontinuities using derivative filters. In the digital image processing developed for ice-microstructure extraction, summarised in Algorithm 1 and detailed below, steps 2.1 and 2.2 belong to the pre-processing, and steps 2.3 and 2.4 correspond to the detection of the discontinuities. Within this general framework, several different procedures can be considered at each step of the algorithm. The algorithm proposed results from such trials at each step. For example, at step 2.1, a "closing then opening" pro- 
Algorithm 1

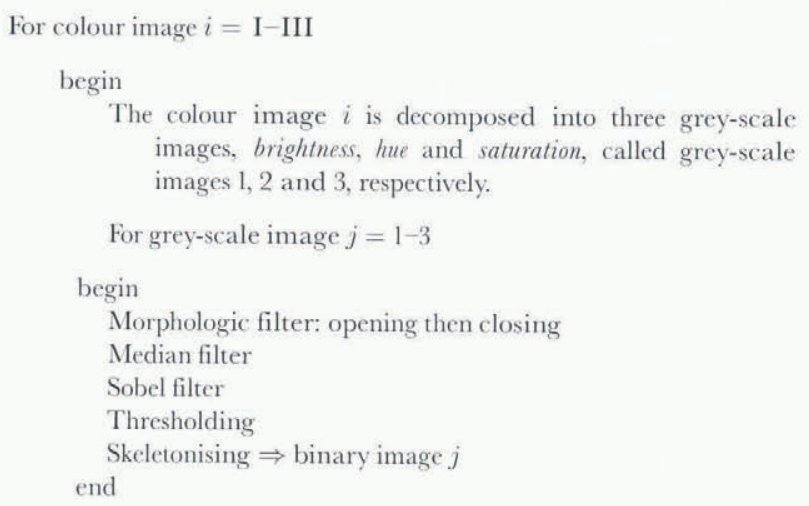

Addition of the three binary images $j \Rightarrow$ binary image $i$ end

Addition of the three binary images $i$

Binary closing: dilation then erosion

Skeletonising

Trim

$\Rightarrow$ microstructure

cedure (instead of "opening then closing") was tested but found to be less efficient in reducing noise (see bclow). Although the proposed image-processing was found to be the most efficient, this does not exclude the possibility of another, completely different, efficient form of processing.

The proposed image segmentation processing is as follows:

1. The first colour image (sce, e.g., Fig. la), denoted I, is decomposed into three grey-scale images, brightness, hue and saturation (Gonzales and Woods, 1992), denoted greyscale images 1, 2 and 3, respectively. Other decompositions of colour images are possible, but this one is chosen because it is used by the human visual system to interpret and differentiate colours. To each pixel of a grey-scale image is assigned an intensity, or grey level, from 0 (black) to 255 (white) and denoted $I[x, y] . x$ and $y$ are the pixel coordinates. The saturation grey-scale image corresponding to Figure la is shown in Figure 2a, as well as the variation of intensity along a section.

2. For each grey-scale image $1-3$, the following steps are performed:

2.1. Morphologic filter. This filter consists of an opening operation, followed by a closing operation. An opening operation is an erosion followed by a dilation, whereas a closing operation is the reverse. Erosion of a grey-scale image consists in assigning to each pixel the minimum intensity of the set of pixels corresponding to this pixel and its neighbours (eight nearest neighbours in the present analysis). Similarly, dilation assigns the maximum intensity of the set to the pixel. More details are given in Gonzales and Woods (1992). This filter reduces highfrequency noise, and allows the removal of insignificant features such as microcracks and pits. The result for a typical image is shown in Figure $2 b$.

2.2. Median filter. The median $m$ of a set of values is such that half the values in the set are $<m$ and half are $>m$. In order to perform median filtering in the neighbour- hood of a pixel, first the intensities $I$ of the pixel and of its neighbours are sorted, then the median is determined and assigned to the pixel. In the present analysis, we limited the calculation to the eight nearest neighbours. This filter reduces noise while preserving the sharpness of the discontinuities. The result is shown in Figure 2c.

2.3. Sobel filter. This filter is a derivative filter which detects discontinuities of intensity. The gradient of intensity (along directions $x$ and $y$ ) is calculated. The maximum value of $\mathrm{d} I / \mathrm{d} x$ and $\mathrm{d} I / \mathrm{d} y$ is assigned to the pixel. This results in low values for homogeneous regions, and very high values along discontinuities (grain boundaries). Thresholding will therefore be easy to perform, in order to extract these discontinuities. More details about Sobel filtering are given in Gonzales and Woods (1992). The image obtained is shown in Figure $2 \mathrm{~d}$ with its associated grey-level histogram.

2.4. Thresholding. A threshold is defined on the histogram (Fig. 2d) by visual testing: the operator "moves" the threshold along the grey scale and selects the best result for the thresholding of boundaries. A grey level of 255 (white) is assigned to the pixels above the threshold, corresponding to discontinuities, and a grey level of 0 (black) is assigned to the pixels below the threshold. This results in a binary image. The three thresholds determined for the three grey-level images (brightness, hue and saturation) are memorised. They will be used for thresholding of the other two colour images, in order to accelerate the process.

2.5. Skeletonising. This operation, also called thinning, determines the skeleton, one pixel wide, of the white regions of the binary image. This classical algorithm is detailed in Gonzales and Woods (1992). The skeleton is shown in Figure 2e.

3. The three binary images resulting from digital processing (steps 2.1-2.5) of the three grey-level images are summed.

4. Operations $1-3$ are repeated for the other two colour images, II and III, using the previously memorised thresholds (see above).

5. The three binary images arising from each of the three colour images I-III are summed. The binary image obtained results from the addition of $3 \times 3=9$ binary images. The result is shown in Figure $2 \mathrm{f}$.

6. Binary closing (dilation then erosion). After step 5, because the three colour images do not coincide perfectly, grain boundaries appear blurred. Binary closing allows blurred boundaries to be reassembled.

\section{Skeletonising.}

8. Final trimming to remove "dead-end" branches.

The microstructure obtained at the end of this algorithm is shown in Figure 1b, to be compared with one of the three starting colour images (Fig. la). Structural and topological parameters can now be computed on this final binary image. The digital image-processing proposed by Eicken (1993), though different, included some operations similar to that employed here, such as grey-scale image opening or Sobel filtering. The order specified in Algorithm 1 is important. If one step is omitted, the quality of image 

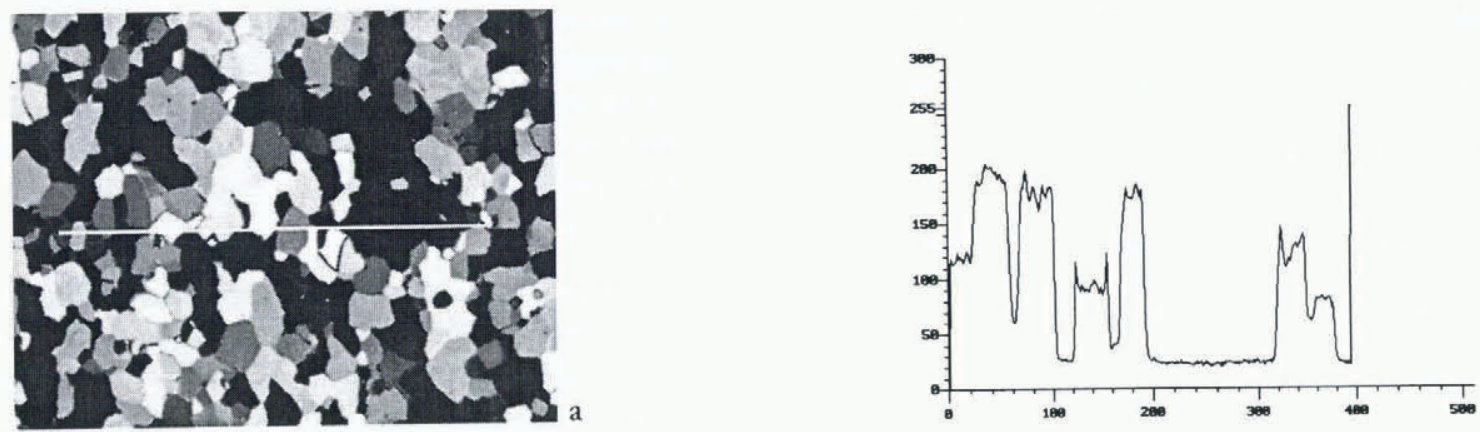

$1 \mathrm{~cm}$
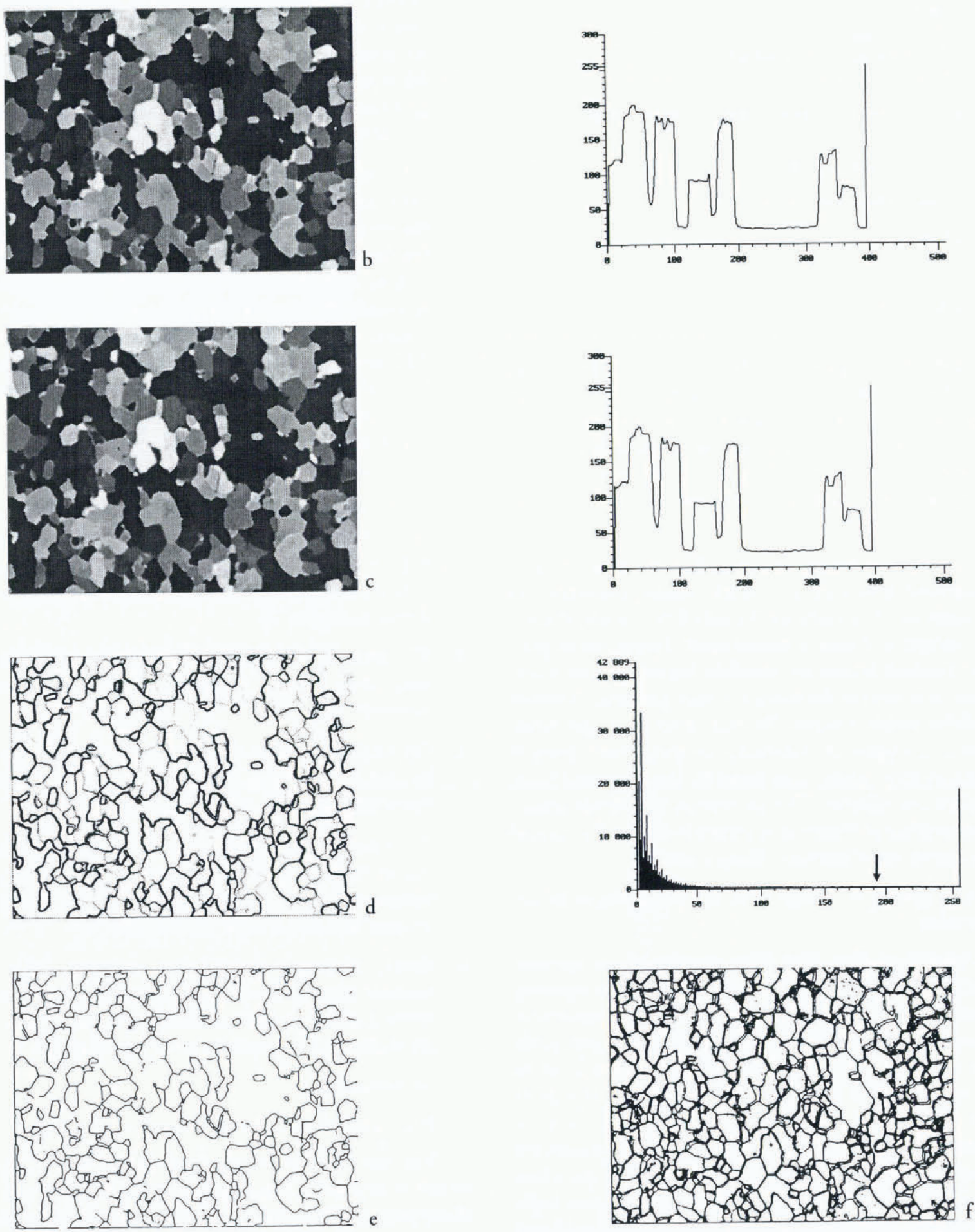

Fig. 2. Successive modifications of a digital image of grains. (a) Saturation grey-scale image of the colour image of Figure la. Left: full image. Right: variation of intensity along a section, indicated by a white line on the left. (b) Grey-scale image after morphologic filter. (c) Grey-scale image after median filter. (d) Grey-scale image after Sobel filter. Right: corresponding greylevel histogram. (e) Binary image after thresholding and skeletonising of the image in $(d)$. $(f)$ Binary image after summation of $3 \times 3=9$ binary images similar to that in (d) (see text for details). 
segmentation decreases and we lose grain boundaries. The morphologic, median and Sobel filters are non-linear filters (Gonzales and Woods, 1992). The use of linear filters was attempted, but it results in a broadening of the transitions (or discontinuities), thus making thresholding more difficult.

An intrinsic limitation of the proposed method is clearly illustrated in Figure lb. When neighbouring grains have very $\operatorname{similar} c$-axis orientations perpendicular to the section, they remain dark on the three colour images and are not easily segmented. This effect, which is particularly pronounced in Figure lb, could be reduced with the help of a universal Rigsby stage. This limitation has little effect on the calculation of mean parameters (e.g. mean area, mean perimeter) but can have an effect on grain-size distribution towards the large sizes. The same limitations apply to manual estimation of grain-sizes. Note that a manual addition of the "obvious" missed boundaries can be performed easily on the computer. Another way to deal with this problem is to remove the most suspicious regions (e.g. regions 1 and 2 in Fig. 1b) from the subsequent statistical analyses.

\section{APPLICATION TO THE EPICA ICE CORE (A PRE- LIMINARY STUDY)}

The EPICA ice-coring started at Dome Concordia $\left(75^{\circ} 06^{\prime} \mathrm{S}, 123^{\circ} 24^{\prime} \mathrm{E}\right.$; $3233 \mathrm{~m}$ a.s.l.) during the austral summer 1996-97, and reached $363 \mathrm{~m}$ depth at the end of the 1997-98 field season. During this season, thin sections of ice were produced along the core at $100-360 \mathrm{~m}$ depth, following the procedure described above, then digitalised and analyzed at the Laboratoire de Glaciologie at Géophysique de l'Environnment (LGGE). Most of them were vertical thin sections about $8 \mathrm{~cm}$ wide and (along the vertical direction) $11 \mathrm{~cm}$ long. Only a fraction of the section, representing a surface of about $27.2 \mathrm{~mm} \times 20.4 \mathrm{~mm}$, was digitalised. At three different depths $(120,218$ and $357 \mathrm{~m})$, three images were digitalised from the same section, but with different image fields, in order to estimate the experimental scatter on the structural parameters extracted. A few horizontal thin sections were also produced and digitalised at the same resolution.

From the microstructures obtained after image analysis, numerous structural and topological parameters can be extracted in order to characterise the material. To illustrate the potential of the procedure described above, we will now focus on three problems: the definition of grain "size", and its influence on grain-growth kinetics; shape anisotropy; and morphology of grains.

\section{Mean grain-size and grain growth}

Like many crystalline materials (see, e.g., Ralph, 1990) at elevated relative temperature (at least $T / T_{\mathrm{F}}>0.5$ ), polar ice experiences grain growth through time. The driving force for grain growth arises from a reduction of the total grainboundary free energy within the system (Duval and Lorius, 1980; Ralph, 1990). Normal grain growth, i.e. a linear increase of the mean cross-sectional area, $\bar{A}$, with time $t$ (Alley and Woods, 1996), has been reported for shallow ice of cold ice sheets (Gow and Williamson, 1976; Duval and Lorius, 1980).

$$
\bar{A}=\bar{A}_{0}+K t,
$$

where $\bar{A}_{0}$ is the initial mean cross-sectional area and $K$ is a constant showing an Arrhenius dependence on tempera- ture. This was sometimes expressed as a parabolic growth law for the mean grain "size" $\bar{d}$ :

$$
\bar{d}^{2}=\bar{d}_{0}^{2}+K t .
$$

It is not the purpose of the present work to discuss the validity, the physical or climatic significance of such a growth law (see Alley and others, 1986; Petit and others, 1987). We will restrict our analysis to the relationship between grain "size", $d$, and grain cross-sectional area, $A$, and to the influence of the method of size estimation on subsequent growth law.

Figure 3 shows the evolution of the mean squared grainsize, $\bar{d}^{2}$, with depth for the EPICA ice core, $\bar{d}^{2}$ being estimated by a different method. The first method (squares) corresponds to the averaging of cross-sectional area of grains, $\bar{A}$, easily obtained from image analysis of an ice-microstructure map such as that shown in Figure 1b. Circles represent the mean cross-sectional area of the 50 largest grains in each section, $\bar{A}_{50}$, a way of estimating grain-size similar to that employed by Gow (1969). Triangles correspond to the square of the mean linear intercept length, $\bar{L}^{2}$. At the three depths where three different images were digitalised from the same sections, the corresponding different estimations of the mean cross-sectional area fall within a range of about $0.30 \mathrm{~mm}^{2}$, whatever the method used $\left(\bar{A}, \bar{A}_{50}\right.$ or $\left.\bar{L}^{2}\right)$. Estimation of $\bar{L}$ was performed automatically with lines along the vertical and horizontal directions and a line crossing the image each two pixels. This obviously represents a much larger line density than those used for manual measures, thus reducing scatter. These three differ-

\section{Mean cross-sectional area $\left(\mathrm{mm}^{2}\right)$}

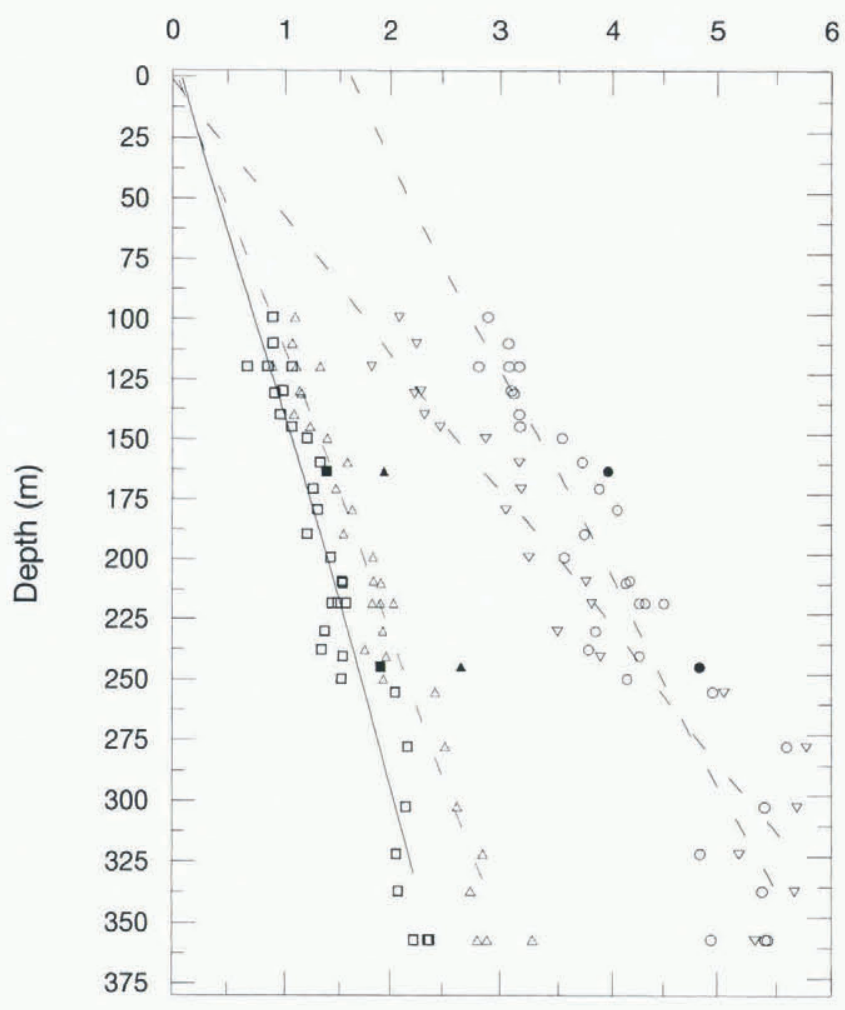

Fig. 3. Evolution of the mean cross-sectional area of grains with depth from the shallow part of the EPICA ice core. Squares: deduced directly from image analysis of ice microstructure. Triangles: deduced from the linear intercept method. Circles: mean cross-sectional area of the 50 largest grains. Inverted triangles: mean cross-sectional area of the largest $25 \%$ of grains. Open symbols: vertical thin sections. Filled symbols: horizontal thin sections. 
ent datasets are well described by linear fits, but with different slopes. For shallow ice of large ice sheets, a linear relationship between depth and age, $t$, is a very reasonable approximation. Therefore, the Figure 3 data seem to support Equation (1). However, the constant $K$ varies with the method used to evaluate the mean squared grain-size up to a factor of about 2 (see Table 1). Moreover, if one extends the linear fit for $\bar{A}_{50}$ up to the surface $(0 \mathrm{~m})$, a much larger "initial" value is found compared to the $\bar{A}$ or $\bar{L}^{2}$ methods. This results from a bias introduced by a representativity evolving with mean grain-size (see above). The relationship between $\bar{A}$ and the mean cross-sectional area of the 50 largest grains, $\bar{A}_{50}$, is not trivial and obviously depends on size distribution, which could change over time and from site to site (because of temperature and climatic differences). During normal grain growth, the shape of the grain-size distribution is not supposed to change, and some authors have proposed log-normal distributions to describe experimental data or results of computer simulation (see, e.g., Atkinson, 1988; Anderson and others, 1989). Such a distribution, however, so far has no theoretical basis. Moreover, the peak value and the standard deviation of the distribution change over time. Therefore, an estimation of activation energy for grain growth by comparison of grain-growth kinetics, deduced from the $\bar{A}_{50}$ method, at different sites with different mean annual temperatures (see, e.g., Gow, 1969), has to be made with caution. As mentioned above, an additional problem of the $\bar{A}_{50}$ method comes from the increasing representativity of the 50 largest grains as the mean grain-size increases. In the present analysis, this representativity increased from about $10 \%$ at $100 \mathrm{~m}$ to $25 \%$ at $360 \mathrm{~m}$, which is less than in the Gow (1969) analysis $(\geq 25 \%)$. To examine a possible effect of such increasing representativity, we computed the mean cross-sectional area of the largest $25 \%$ of grains in each section, $\bar{A}_{25 \%}$ (see Fig. 3). With this method, the extrapolation of the linear fit up to $0 \mathrm{~m}$ is very close to that found with the $\bar{A}$ or $\bar{L}^{2}$ methods (see Table 1), and $75 \%$ of the available information is still lost.

In classical normal grain-growth analytical models (two-dimensional models; see, e.g., Hillert, 1965, adapted by Alley and others, 1986 for ice; Atkinson, 1988), the critical size parameter, $d_{\text {crit }}$ or $A_{\text {crit }}$, such that grains larger than $A_{\text {crit }}$ grow and smaller grains shrink, is equal to $\bar{A}$ (or $\bar{d}$ ). Any other value of $A$, including the mean cross-sectional area of the 50 largest grains, has much less physical significance with respect to theoretical models. However, three-dimensional parameters such as the mean grain volume would have even more physical meaning. From three-dimensional computer simulations of normal grain growth, Anderson and others (1989) showed that, if the morphology of grains is such that grain shapes are compact and exhibit close to minimal surface areas with respect to grain volume at all times, then the "true" grain-growth kinetics, based on the evolution of the mean grain volume, is very close to the kinetics derived from two-dimensional parameters (mean cross-sectional area, $\bar{A}$ ). In particular, the same constant $K$ is found (Anderson and others, 1989). This similitude does not hold if morphology is changing over time. To the authors' knowledge, the only available dataset on the distribution of grain volumes and corresponding cross-sections and linear intercepts for a "realistic" microstructure is Anderson and other (1989). On this (limited) basis, we argue that the mean cross-sectional area method $(\bar{A})$ is the most "exact" for deriving true three-dimensional grain-growth kinetics from thin-section analyses. In Table 1 , the constant $K$ obtained with the 50 largest grains method is close to that obtained by Duval and Lorius (1980) from a former core drilled at the "old" Dome C site $\left(74^{\circ} 39^{\prime} \mathrm{S}, 124^{\circ} 10^{\prime} \mathrm{E}\right.$, about $70 \mathrm{~km}$ from the current Dome Concordia drilling site), in agreement with the method used by these authors to estimate grain-sizes.

The linear intercept method gives results closer to $\bar{A}$ than the 50 largest grains method. However, for a given volume element (a "grain"), the relation between $\bar{A}$ and $\bar{L}$ depends on the element morphology (Underwood, 1970). The ratio $\bar{A} / \bar{L}^{2}$ is equal to $3 \pi / 8 \approx 1.18$ for a sphere. For a truncated octahedron, a volume element allowing space filling and sometimes used to model grains (Gibson and Ashby, 1988), this ratio is about 1.32 . For a space-filling assembly of grains of different sizes, isotropic on average, $\bar{L}$ depends on both $\bar{A}$ and the mean perimeter of grains, $\bar{s}$ (Underwood, 1970):

$$
\bar{L}=\pi \frac{\bar{A}}{\bar{s}} .
$$

For a grain assembly with shape anisotropy, the problem is even more complex. It is shown below that the average shape anisotropy of grains varies with depth (and so time) along the EPICA ice core. The linear intercept method is therefore unable to exactly reproduce the evolution of $\bar{A}$ and thus the evolution of the mean grain volume, as shown by Anderson and others (1989).

From the above discussion, it appears that in two dimensions the measure of the mean cross-sectional area, $\bar{A}$, is the size definition $\left(\bar{d} \sim \bar{A}^{1 / 2}\right)$ with the best physical significance. This measure is difficult to determine manually, a fact which shows the relevance of the automatic procedure described here.

Finally, one can notice in Figure 3 that the mean crystal sizes observed on horizontal sections are systematically slightly larger than expected from the regression analysis, whatever the definition of the size. This results from the flattening of grains on the horizontal plane (see below).

\section{Shape anisotropy}

In order to show a possible anisotropy of grain shape, the ratio between the mean linear intercepts in the horizontal $(X)$ and vertical $(Z)$ directions, $\bar{L}_{X} / \bar{L}_{Z}$, has been cal-

Table 1. Grain-growth kinetics for the shallow part (Holocene ice) of the EPICA ice core, deduced from different grain-size definitions (see text for details). Grain-growth kinetics are expressed in terms of mean squared grain-size vs depth: $\bar{d}^{2}=\bar{d}_{0}^{2}+K^{\prime} z$, where $z$ is depth ( $m$ )

\begin{tabular}{|c|c|c|c|c|c|}
\hline $\begin{aligned} & K^{\prime}\left(\mathrm{mm}^{2} \mathrm{~m}^{-1}\right) \\
R^{2} & \text { (regression coeff. })\end{aligned}$ & $\begin{array}{c}0.60 \times 10^{-2} \\
0.92\end{array}$ & $\begin{array}{c}0.80 \times 10^{-2} \\
0.95\end{array}$ & $\begin{array}{c}1.00 \times 10^{-2} \\
0.86\end{array}$ & $\begin{array}{c}1.68 \times 10^{-2} \\
0.93\end{array}$ & $1.10 \times 10^{-2}$ \\
\hline
\end{tabular}




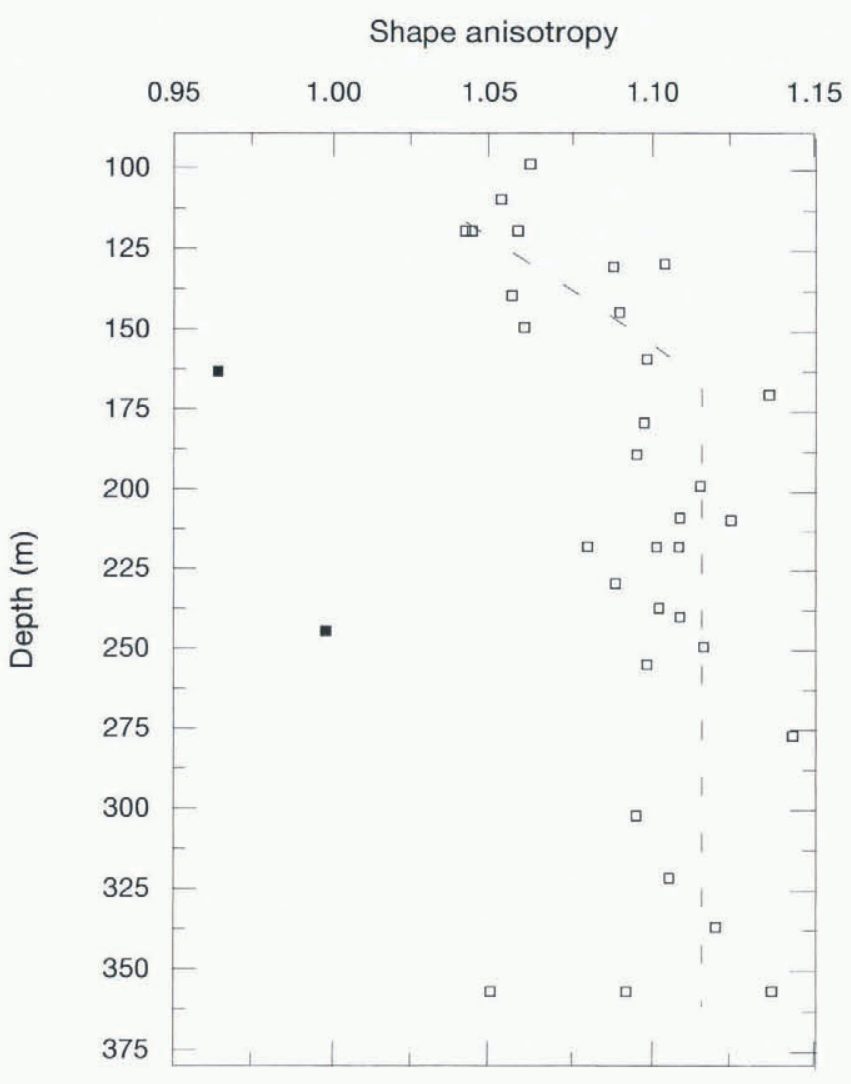

Fig. 4. Evolution of shape anisotropy with depth from the shallow part of the EPICA ice core. Triangles: vertical thin sections; anisotropy measured by $\bar{L}_{X} / \bar{L}_{Z}$ (Z: vertical direction, $X$ : horizontal direction). Squares: horizontal thin sections; anisotropy measured by $\bar{L}_{\mathrm{X}} / \bar{L}_{\mathrm{Y}}(X$ and $Y$ : horizontal directions).

culated on vertical thin sections (Fig. 4). This ratio is systematically larger than 1, which means that the grains are flattened along the horizontal plane. Flattening seems to increase with depth, from about 1.05 at $100 \mathrm{~m}$ to about 1.12 at $180-200 \mathrm{~m}$, then remains roughly constant up to $360 \mathrm{~m}$. At the three depths where three different images were digitalised from the same sections, the different estimations of $\bar{L}_{X} / \bar{L}_{Z}$ fall within a range of about $0.03-0.04$, except for one image at $357 \mathrm{~m}$ which exhibits a surprisingly low flattening. On the other hand, grains are isotropic on horizontal thin sections, with a ratio $\bar{L}_{X} / \bar{L}_{Y}$, close to 1 ( $Y$ is a second horizontal direction). Such a shape anisotropy makes the link between $\bar{L}$ and $\bar{A}$ even more difficult to establish (see above).

\section{Grain morphology}

In Figure 5 is plotted the evolution of the ratio $A / s^{2}$ (crosssectional area/perimeter ${ }^{2}$ ) averaged over all the grains of the section, with depth. This adimensional mean form factor, which is maximum for spheres $\left(A / s^{2}=1 / 4 \pi \approx 0.08\right)$, is a measure of the average grain morphology. The latter is remarkably stable during grain growth, around a value of 0.055. This suggests that grain morphologies are close, on average, to an equilibrium-minimising surface area (in three dimensions) with respect to grain volume, while subject to topological constraints and flattening.

\section{GONCLUSION AND PROSPEGT}

An automatic procedure, based on colour-image analysis of

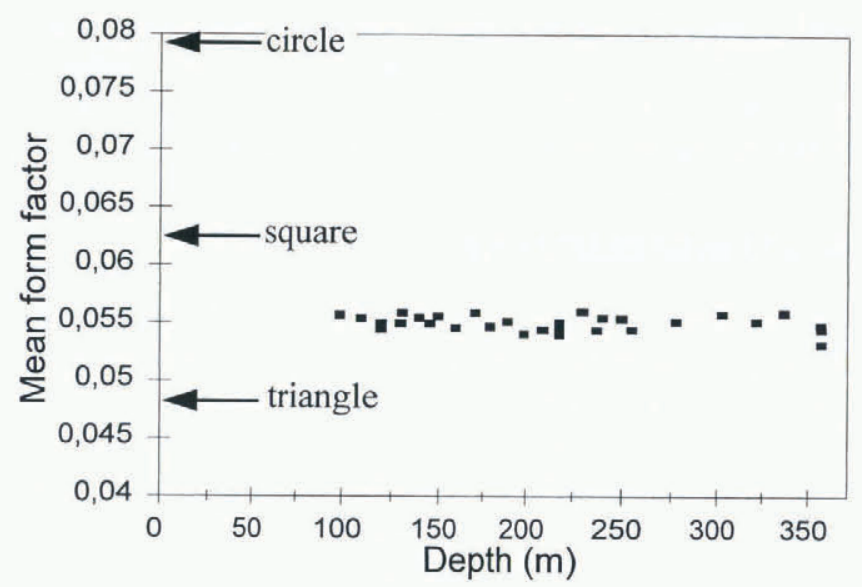

Fig. 5. Evolution of the average grain morphology, expressed by the form factor $A / s^{2}$ averaged over all the grains of the section, with depth, for the shallow part of the EPICA ice core. Arrows indicate, for comparison, the form factor of three simple two-dimensional shapes (circle, square and equilateral triangle).

thin sections observed between crossed polarisers, has been developed in order to extract two-dimensional (cross-sectional) ice microstructure. From these microstructures, numerous structural, morphological and topological parameters can be determined to characterise the material and its evolution. This has been illustrated with three examples on the shallow ice of the EPICA ice core, including mean cross-sectional area of grains, shape anisotropy and average grain morphology.

However, averaged values of parameters are not sufficient to follow in detail the evolution of the microstructure. For example, the evolution of grain-size distribution through time can be used to differentiate grain-growth regimes (normal vs abnormal; see, e.g., Hillert, 1965; Ralph, 1990). Statistical distributions of parameters can be extracted easily and automatically from microstructures such as that represented in Figure lb. Arnaud (1997) obtained such distributions for Vostok firn, using a different technique to reveal the microstructure (see above).

Topological parameters (e.g. the number of sides per grain (in two dimensions)), are also important for characterising the physics of polycrystalline materials (Atkinson, 1988), including ice (Arnaud, 1997). Topological two-dimensional parameters are easily obtained from reconstructed ice microstructures.

The ultimate goal of the study of ice microstructure is to infer three-dimensional parameters, such as volumes of grains, from two-dimensional parameters obtained on cross-section. This is a difficult problem. As noted above, in the case of isotropic microstructures and compact grain shapes, mean grain volume could be correctly estimated from the mean cross-sectional area (Anderson and others, 1989). This is no longer true for anisotropic microstructures or changing morphologies. Thorvaldsen (1997) stressed that two polycrystals with identical mean linear intercepts on a two-dimensional section can have different mean grain volumes, depending on grain-volume distribution and grain shapes. The correspondence between cross-sectional area distributions and grain-volume distributions is even less straightforward (Anderson and others, 1989). A complete description of the two-dimensional microstructure, and especially statistical distributions of structural and topological parameters, is necessary, though perhaps not sufficient, 
and simplifying stereological hypotheses on isotropy and morphology are required in order to infer three-dimensional parameters. Such an analysis would be impossible to perform from manual analysis of thin sections.

\section{ACKNOWLEDGEMENTS}

This work is a contribution to the "European Project for Ice Coring in Antarctica" (EPICA), a joint European Science Foundation/European Commission scientific programme, funded by the European Commission under Environment and Climate Programme (1994-98) contract ENV4-CT950074 and by national contributions from Belgium, Denmark, France, Germany, Italy, The Netherlands, Norway, Sweden, Switzerland and the U.K.

We would like to thank P. Duval and L. Arnaud for valuable discussions and comments, as well as two anonymous reviewers whose comments and suggestions helped to improve the clarity of the paper. LGGE is associated with Université Joseph Fourier, Grenoble I.

\section{REFERENCES}

Alley, R. B. and G. A. Woods. 1996. Impurity influence on normal grain growth in the GISP2 ice core, Greenland. F. Glaciol., 42(141), 255-260.

Alley, R. B., J. H. Perepezko and C. R. Bentley. 1986. Grain growth in polar ice: I. Theory. 7. Glaciol., 32(112), 415-424.

Anderson, M. P., G. S. Grest and D. J. Srolovitz. 1989. Computer simulation of normal grain growth in three dimensions. Philos. Mag. B, 59 (3), 293-329.
Arnaud, L. 1997. Modélisation de la transformation de la neige en glace à la surface des calottes polaires: étude du transport des gaz dans ces milieux poreux. (Thèse de doctorat, Université Joseph Fourier, Grenoble I.

Arnaud, L., M. Gay, J.-M. Barnola and P. Duval. 1998. Imaging of firn and bubbly ice in coaxial reflected light: a new technique for the characterization of these porous media. F. Glaciol., 44(147), 326-332.

Atkinson, H.V. 1988. Theories of normal grain growth in pure single phase systems. Acta Metall., 36(3), 469-491.

Duval, P. and C. Lorius. 1980. Crystal size and climatic record down to the last ice age from Antarctic ice. Earth Planet. Sci. Lell., 48(1), 59 - 64.

Eicken, H. 1993. Automated image analysis of ice thin sections - instrumentation, methods and extraction of stereological and textural parameters. 7. Glaciol., 39(132), 341-352.

Gibson, L. J. and M. F. Ashby. 1987. Cellular solids: structure and properties. New York, Pergamon Press.

Gonzales, R. C. and R. E. Woods. 1992. Digital image processing. Reading, MA, Addison-Wesley Publishing Co.

Gow, A. J. 1969. On the rates of growth of grains and crystals in South Polar firn. 7. Glaciol., 8(53), 241-252.

Gow, A. J. and T. Williamson. 1976. Rheological implications of the internal structure and crystal fabrics of the West Antarctic ice sheet as revealed by deep core drilling at Byrd Station. Geol. Soc. Am. Bull., 87(12), 1665-1677.

Hillert, M. 1965. On the theory of normal and abnormal grain growth. Acta Metall., 13, 227-238.

Petit, J. R., P. Duval and C. Lorius. 1987. Long-term climatic changes indicated by crystal growth in polar ice. Nature, 326 (6108), 62-64.

Ralph, B. 1990. Grain growth. Mater. Sci. Tech., 6, 1139-1144.

Thorsteinsson, Th., J. Kipfstuhl, H. Eicken, S.J. Johnsen and K. Fuhrer. 1995. Crystal size variations in Eemian-age ice from the GRIP ice core, central Greenland. Earth Planet. Sci. Lett., 131 (3-4), 381-394.

Thorvaldsen, A. 1997. The intercept method - 2. Determination of spatial grain size. Acta Materialia, 45 (2), 595-600.

Underwood, E. E., ed. 1970. Quantitative stereology. Reading, MA, AddisonWesley Publishing Co.

MS received 23 November 1998 and accepted in revised form 14 May 1999 